\title{
Lag Synchronization of Coupled Delayed Chaotic Neural Networks by Periodically Intermittent Control
}

\author{
Songjian Dan, ${ }^{1,2}$ Simon X. Yang, ${ }^{1,3}$ and Wei Feng ${ }^{4}$ \\ ${ }^{1}$ College of Communication Engineering, Chongqing University, Chongqing 400030, China \\ ${ }^{2}$ School of Engineering, University of Guelph, Guelph, ON, Canada N1G 2 W1 \\ ${ }^{3}$ College of Continuing Education, Chongqing University of Education, Chongqing 400065, China \\ ${ }^{4}$ School of Mathematics and Information Engineering, Chongqing University of Education, Chongqing 400065, China
}

Correspondence should be addressed to Songjian Dan; dsjian@163.com

Received 17 October 2012; Accepted 30 December 2012

Academic Editor: Chuandong Li

Copyright (C) 2013 Songjian Dan et al. This is an open access article distributed under the Creative Commons Attribution License, which permits unrestricted use, distribution, and reproduction in any medium, provided the original work is properly cited.

We investigate the lag synchronization of coupled neural networks with time delay. Some sufficient conditions for lag synchronization will be derived by using Lyapunov stability theory and intermittent control. Compared to existing results, some less conservative conditions are derived to guarantee the stabilization of error system. The analytical results are confirmed by numerical simulations.

\section{Introduction}

After the seminal works of Pecora and Carroll [1], the idea of synchronization of chaotic systems has received a great deal of interest among researchers from various fields. Over the past decades, several different regimes of chaos synchronization have been investigated, for example, complete synchronization $[1,2]$, generalized synchronization [3], projective synchronization [4], phase synchronization [5], lag synchronization [6], and anticipating synchronization [7]. In recent years, Wen et al. have studied the stability analysis and synchronization of the memristor-based recurrent neural networks $[8,9]$. On the other hand, it has been shown that the complete synchronization of chaos is practically impossible for the finite speed of signals. Chaotic lag synchronization appears as a coincidence of shift-in-time states of interactive systems. It is just synchronization lag that makes lag synchronization practically available. For instance, in the telephone communication system, the voice one hears on the receive side at time $t$ is often the voice from the transmitter side at time $t-\tau$ [10]. So, in many cases, it is more reasonable to require the slave system to synchronize the master system with a time delay $\tau$. Thus, it is of great importance to study lag synchronization and some results have been reported in this research area. Shahverdiev et al. [11] investigated lag synchronization between unidirectional coupled delayed Ikeda systems by using feedback control techniques. Li et al. [12] considered lag synchronization problem with an application in secure communication. Zhou et al. [13] studied lag synchronization of coupled chaotic delayed neural networks by using adaptive feedback control techniques.

In this paper, we have formulated the lag synchronization problem for chaotic neural networks by means of periodically intermittent control. Our interest focuses on the class of intermittent control with time duration; namely, the control is activated in certain nonzero time intervals and inactivated in other time intervals. Also, we will remove this limitation and design a general periodically intermittent controller for chaotic neural networks.

The rest of the paper is organized as follows. In Section 2, we formulate the problem of lag synchronization of coupled 
systems. In Section 3, a general convergence criterion for the lag synchronization error of coupled systems is established. Also, some corollaries and remarks are listed to show the advantage of this paper. In Section 4, numerical examples are given to show the theoretical results, which is followed by the conclusions in Section 5.

\section{Problem Formulation and Preliminaries}

In this paper, we consider the chaotic time-delay systems described by

$$
\begin{gathered}
\dot{x}(t)=C x(t)+A f(x(t))+B g(x(t-\tau)), \quad t>0, \\
x(t)=\varphi(t), \quad-\tau \leq t \leq 0,
\end{gathered}
$$

where $x \in R^{m}$ denotes the state vector, $C, A$, and $B \in R^{m \times m}$ are constant matrices, $\tau$ is the time delay, $f, g: R^{m} \rightarrow R^{m}$ are nonlinear functions satisfying the Lipschitz condition; namely, there exist positive constants $L_{f}, L_{g}$ such that

$$
\begin{gathered}
|f(\alpha)-f(\beta)| \leq L_{f}|\alpha-\beta|, \\
|g(\alpha)-g(\beta)| \leq L_{g}|\alpha-\beta|, \quad \forall \alpha, \beta \in R^{n} .
\end{gathered}
$$

In order to lag-synchronize system (1) by means of periodically intermittent feedback control, we assume that the corresponding slave system as

$$
\begin{gathered}
\dot{y}(t)=C y(t)+A f(y(t))+B g(y(t-\tau))+u(t), \quad t>0, \\
y(t)=\psi(t), \quad-\tau \leq t \leq 0,
\end{gathered}
$$

where $y \in R^{m}$ denotes the state vector, and $u(t)$ is the intermittent linear state feedback control gain defined as follows:

$$
u(t)= \begin{cases}k(x(t-\theta)-y(t)), & n T \leq t<n T+\sigma T \\ 0, & n T+\sigma T \leq t<(n+1) T\end{cases}
$$

where $k$ denotes control strength, $0<\sigma<1$ denotes switching rate, $T$ denotes control period, and $\theta$ is the transmittal delay.

Defining the lag synchronization error between the systems (1) and (3) as $e(t)=y(t)-x(t-\theta)$, we have the following error dynamical system:

$$
\begin{aligned}
\dot{e}(t)= & \dot{y}(t)-\dot{x}(t-\theta) \\
= & C e(t)+A(f(y(t))-f(x(t-\theta))) \\
& +B(g(y(t-\tau))-g(x(t-\tau-\theta)))+u(t) .
\end{aligned}
$$

Under the control of the form (4), the system (5) can be rewritten as

$$
\begin{gathered}
\dot{e}(t)=(C-k I) e(t)+A(f(y(t))-f(x(t-\theta))) \\
+B(g(y(t-\tau))-g(x(t-\tau-\theta))), \\
n T \leq t<n T+\sigma T, \\
\dot{e}(t)=C e(t)+A(f(y(t))-f(x(t-\theta))) \\
+B(g(y(t-\tau))-g(x(t-\tau-\theta))), \\
n T+\sigma T \leq t<(n+1) T .
\end{gathered}
$$

In order to define the initial condition of system (6), we supplement the state $x(t)$ on $[-\theta-\tau,-\tau]$ as

$$
x(t)=\varphi(-\tau), \quad-\tau-\theta \leq t<-\tau .
$$

Introduce a new notation $\varphi(t)$ as

$$
\widehat{\varphi}(t)= \begin{cases}\varphi(t), & -\tau \leq t \leq 0, \\ \varphi(-\tau), & -\tau-\theta \leq t \leq-\theta .\end{cases}
$$

Then initial condition of system (6) is defined by

$$
e(t)=\psi(t)-\varphi(t-\theta), \quad-\tau \leq t \leq 0 .
$$

To establish the main result of this paper, the following preliminaries are necessary.

Lemma 1 (see [14]). Given any real matrices $\Sigma_{1}, \Sigma_{2}, \Sigma_{3}$ of appropriate dimensions and a scalar $s>0$, such that $0<\Sigma_{3}=$ $\Sigma_{3}^{T}$. Then the following inequality holds:

$$
\Sigma_{1}^{T} \Sigma_{2}+\Sigma_{2}^{T} \Sigma_{1} \leq s \Sigma_{1}^{T} \Sigma_{3} \Sigma_{1}+s^{-1} \Sigma_{2}^{T} \Sigma_{3}^{-1} \Sigma_{2} .
$$

Lemma 2 (Halanay inequality [15]). Let $\omega:[\mu-\tau, \infty) \rightarrow[0$, $\infty)$ be a continuous function such that

$$
\dot{\omega}(t) \leq-a \omega(t)+b \max \omega_{t}
$$

is satisfied for $t \geq u$. If $a>b>0$, then

$$
\omega(t) \leq\left[\max \omega_{\mu}\right] \exp \{-\gamma(t-\mu)\}, \quad t \geq \mu,
$$

where $\max \omega_{t}=\sup _{t-\tau \leq \theta \leq t} \omega(\theta)$, and $\gamma>0$ is the smallest real root of the equation

$$
a-\gamma-b \exp \{\gamma \tau\}=0 .
$$

Lemma 3 (see [16]). Let $\omega$ be a nonnegative function defined on the interval $\left[t_{0}-\tau, \infty\right)$ and continuous on the subinterval $\left[t_{0}, \infty\right)$. Assume that

$$
\dot{\omega}(t) \leq a \omega(t)+b \omega(t-\tau)
$$

is satisfied for $t \geq t_{0}$. If $a>0, b>0$, then

$$
\omega(t) \leq \bar{\omega}_{t_{0}} \exp \left\{\eta\left(t-t_{0}+\tau\right)\right\}, \quad t \geq t_{0},
$$

where $\bar{\omega}_{t_{0}}=\sup _{t_{0}-\tau \leq \theta \leq t_{0}} \omega(\theta)$ and $\eta>0$ is the unique root of the equation $a+b e^{-\eta \tau}=\eta$. 


\section{Main Results}

In this section, we shall stabilize lag synchronization error system based on Lyapunov method and the intermittent control technique.

Theorem 4. Suppose that there exist positive scalars $h_{1}>0$, $h_{2}>0$, and $s_{i}>0(i=1,2,3,4,5,6)$ such that

(i) $C+C^{T}-2 k I+s_{1} A A^{T}+s_{1}^{-1} L_{f}^{2} I+s_{2} B B^{T}+h_{1} I \leq 0$,

(ii) $C+C^{T}+s_{3} A A^{T}+s_{3}^{-1} L_{f}^{2} I+s_{4} B_{2} B_{2}^{T}-h_{2} I \leq 0$,

(iii) $h_{1}>s_{2}^{-1} L_{g}^{2}$, and $\gamma(\sigma T-\tau)-\eta(T-\sigma T+\tau)>0$, where $\gamma$ is the unique positive root of the equation $h_{1}-\gamma-$ $s_{2}^{-1} L_{g}^{2} \exp \{\gamma \tau\}=0$. Then, error system (6) is globally exponentially stable; that is, the master system (1) and the slave system (3) achieve lag synchronization.

Proof. Consider the following Lyapunov function:

$$
V(t)=e(t)^{T} e(t) .
$$

When $n T \leq t<n T+\sigma T$, the derivative of (16) with respect to time $t$ along the trajectories of the first subsystem of the system (6) is calculated and estimated as follows:

$$
\begin{aligned}
\dot{V}(t)=2 e(t)^{T} \dot{e}(t) & \\
=2 e(t)^{T}[(C-k I) e(t) & \\
& +A(f(y(t))-f(x(t-\theta))) \\
& +B(g(y(t-\tau))-g(x(t-\tau-\theta)))] \\
= & e(t)^{T}\left(C^{T}+C-2 k I\right) e(t) \\
& +2 e(t)^{T} A(f(y(t))-f(x(t-\theta))) \\
& +2 e(t)^{T} B(g(y(t-\tau))-g(x(t-\tau-\theta))) .
\end{aligned}
$$

Using Lemma 1, we have the following estimation:

$$
\begin{array}{rl}
2 e(t)^{T} & A(f(y(t))-f(x(t-\theta))) \\
\leq & s_{1} e(t)^{T} A A^{T} e(t)+s_{1}^{-1}(f(y(t))-f(x(t-\theta)))^{T} \\
& \times(f(y(t))-f(x(t-\theta))) \\
\leq & s_{1} e(t)^{T} A_{2} A_{2}^{T} e(t)+s_{1}^{-1} L_{f}^{2} e(t)^{T} e(t) \\
= & e(t)^{T}\left[s_{1} A_{2} A_{2}^{T}+s_{1}^{-1} L_{f}^{2} I\right] e(t) .
\end{array}
$$

Similarly, we have the following estimation for another term:

$$
\begin{aligned}
& 2 e(t)^{T} B(g(y(t-\tau))-g(x(t-\tau-\theta))) \\
& \quad \leq s_{2} e(t)^{T} B B^{T} e(t)+s_{2}^{-1} L_{g}^{2} e(t-\tau)^{T} e(t-\tau) .
\end{aligned}
$$

Substituting these into (17), we have the following:

$$
\begin{aligned}
\dot{V}(t) \leq & e(t)^{T}\left(C+C^{T}-2 k I\right) e(t) \\
& +e(t)^{T}\left[s_{1} A A_{2}^{T}+s_{1}^{-1} L_{f}^{2} I\right] e(t) \\
& +s_{2} e(t)^{T} B B^{T} e(t) \\
& +s_{2}^{-1} L_{g}^{2} e(t-\tau)^{T} e(t-\tau) \\
= & e(t)^{T}\left[C+C^{T}-2 k I+s_{1} A A^{T}\right. \\
& \left.\quad+s_{1}^{-1} L_{f}^{2} I+s_{2} B B^{T}+h_{1} I\right] e(t) \\
& -h_{1} e(t)^{T} e(t)+s_{2}^{-1} L_{g}^{2} e(t-\tau)^{T} e(t-\tau) \\
\leq & -h_{1} e(t)^{T} e(t)+s_{2}^{-1} L_{g}^{2} e(t-\tau)^{T} e(t-\tau) \\
\leq & -h_{1} V(t)+s_{2}^{-1} L_{g}^{2} V(t-\tau) .
\end{aligned}
$$

Similarly, when $n T+\sigma T \leq t<(n+1) T$, one obtains

$$
\begin{aligned}
\dot{V}(t)= & 2 e(t)^{T} \dot{e}(t) \\
= & 2 e(t)^{T}[C e(t)+A(f(y(t))-f(x(t-\theta))) \\
& \quad+B(g(y(t-\tau))-g(x(t-\tau-\theta)))] \\
\leq & e(t)^{T}\left(C+C^{T}\right) e(t) \\
& +e(t)^{T}\left[s_{3} A A^{T}+s_{3}^{-1} L_{f}^{2} I\right] e(t) \\
& +s_{4} e(t)^{T} B B^{T} e(t)+s_{4}^{-1} L_{g}^{2} e(t-\tau)^{T} e(t-\tau) \\
= & e(t)^{T}\left[C+C^{T}+s_{3} A A^{T}\right. \\
& \left.\quad+s_{3}^{-1} L_{f}^{2} I+s_{4} B B^{T}-h_{2} I\right] e(t) \\
& +h_{2} e(t)^{T} e(t)+s_{4}^{-1} L_{g}^{2} e(t-\tau)^{T} e(t-\tau) \\
\leq & h_{2} e(t)^{T} e(t)+s_{4}^{-1} L_{g}^{2} e(t-\tau)^{T} e(t-\tau) \\
\leq & h_{2} V(t)+s_{4}^{-1} L_{g}^{2} V(t-\tau) .
\end{aligned}
$$

Therefore,

$$
\begin{array}{r}
\dot{V}(t) \leq-h_{1} V(t)+s_{2}^{-1} L_{g}^{2} V(t-\tau), \\
n T \leq t<n T+\sigma T ; \\
\dot{V}(t) \leq h_{2} V(t)+s_{4}^{-1} L_{g}^{2} V(t-\tau), \\
n T+\sigma T \leq t<(n+1) T .
\end{array}
$$


By Lemmas 2 and 3, following the same line of argument of the proof of Theorem 1 of [16], we can obtain

$$
\begin{aligned}
\|x(t)\|^{2} & =V(x(t)) \\
& \leq \bar{\omega}(0) \exp \left\{-[\gamma(\sigma T-\tau)-\eta(T-\sigma T+\tau)] \frac{t}{T}\right\},
\end{aligned}
$$$$
\text { for any } t \geq 0 \text {. }
$$

This implies that the error system (6) is globally exponentially stable, and the following estimate holds:

$\|x(t)\|$

$$
\leq|\phi| \exp \left\{-\frac{1}{2}[\gamma(\sigma T-\tau)-\eta(T-\sigma T+\tau)] \frac{t}{T}\right\}, \quad t \geq 0 .
$$

The proof is thus completed.

For computational purpose, we now present a numerically tractable lag synchronization conditions. Let

$$
\begin{aligned}
s_{1}= & \sqrt{\frac{L_{f}}{\lambda_{\max }\left(A A^{T}\right)}}, \\
s_{2}= & e^{0.5 \gamma \tau} \sqrt{\frac{L_{g}}{\lambda_{\text {max }}\left(B B^{T}\right)}}, \\
s_{3}= & s_{1}, \\
s_{4}= & e^{-0.5 \eta \tau} \sqrt{\frac{L_{g}}{\lambda_{\max }\left(B B^{T}\right)}}, \\
h_{1}^{*}= & \left(2 k-\lambda_{\max }\left(C+C^{T}\right)\right) \\
& -2 \sqrt{\lambda_{\max }\left(A A^{T}\right) L_{f}}-e^{0.5 \gamma \tau} \sqrt{\lambda_{\max }\left(B B^{T}\right) L_{g}}, \\
h_{2}^{*}= & \lambda_{\max }\left(C+C^{T}\right) \\
& +2 \sqrt{\lambda_{\max }\left(A A^{T}\right) L_{f}}+\sqrt{\lambda_{\max }\left(B B^{T}\right) L_{g}}+1,
\end{aligned}
$$

where $h_{1}^{*} \geq h_{1}, h_{2}^{*} \leq h_{2}$.

One obtains from Theorem 4 the following corollary.

Corollary 5. If there exist constants $\gamma>0, k$ and $\sigma$ satisfying $0<\sigma<1$ such that

$$
\begin{gathered}
k+\lambda_{\max }(C)-\sqrt{\lambda_{\max }\left(A A^{T}\right) L_{f}^{2}} \\
-e^{0.5 \gamma \tau} \sqrt{\lambda_{\max }\left(B B^{T}\right)} L_{g}^{2}-\frac{1}{2} \gamma=0, \\
\gamma(\sigma T-\tau)-\eta(T-\sigma T+\tau)>0,
\end{gathered}
$$

where $\eta>0$ is the root of the equation

$$
\begin{aligned}
& -\lambda_{\max }(C)+\sqrt{\lambda_{\max }\left(A A^{T}\right) L_{f}^{2}} \\
& \quad+e^{-0.5 \eta \tau} \sqrt{\lambda_{\max }\left(B B^{T}\right) L_{g}^{2}}-\frac{1}{2} \eta=0 .
\end{aligned}
$$

Then, the origin of system (6) is globally exponentially stabilized; that is, the master system (1) and the slave system (3) achieve lag synchronization.

Remark 6. Given the period $T$, Corollary 5 allows us to estimate the feasible region $D$ of control parameters $(k, \sigma)$. One observes that the control strength $k$ can be estimated as follows:

$$
\begin{aligned}
k>k^{*}= & -\lambda_{\max }(C)+\sqrt{\lambda_{\max }\left(A A^{T}\right) L_{f}^{2}} \\
& +e^{0.5 \gamma \tau} \sqrt{\lambda_{\max }\left(B B^{T}\right) L_{g}^{2}}+\frac{1}{2} \gamma .
\end{aligned}
$$

From (32), we can estimate the feasible region $D$ of control parameters $(k, \sigma)$

$$
\begin{gathered}
D=\left\{(k, \sigma) \mid k>k^{*}=-\lambda_{\max }(C)+\sqrt{\lambda_{\max }\left(A A^{T}\right) L_{f}^{2}}\right. \\
\left.+e^{0.5 \gamma \tau} \sqrt{\lambda_{\max }\left(B B^{T}\right) L_{g}^{2}}+\frac{1}{2} \gamma, 0<\sigma<1\right\} .
\end{gathered}
$$

\section{Numerical Example}

In this section, we take Lu neural oscillator [17] as an example to show the validity of the proposed results. The programs DDE23 in MATLAB are used to solve numerically the delay differential equations.

Example 7. Consider the Lu neural oscillator described by following delayed differential equations [17]

$$
\dot{x}(t)=-C x(t)+A f(x(t))+B g(x(t-1)),
$$

where

$$
\begin{gathered}
C=\left(\begin{array}{ll}
1 & 0 \\
0 & 1
\end{array}\right), \\
A=\left(\begin{array}{ll}
3.0 & 5.0 \\
0.1 & 2.0
\end{array}\right), \\
B=\left(\begin{array}{cc}
-2.5 & 0.2 \\
0.1 & -1.5
\end{array}\right),
\end{gathered}
$$

and $f(x(t))=g(x(t))=\tanh (x(t))$.

This model was investigated by $\mathrm{Lu}$ in [17] where it is shown to be chaotic as shown in Figure 1. The corresponding slave system is given by

$$
\dot{y}(t)=-C y(t)+A f(y(t))+B g(y(t-1)),
$$

where $f(y(t))=g(y(t))=\tanh (y(t))$.

In this example, one observes that $L_{f}=L_{g}=1$, and $\tau=1$. For numerical simulation, we select $T=8, \sigma=0.8$, $\theta=0.01$, and $k=20$ and plot the lag synchronization error curve as shown in Figure 2. 


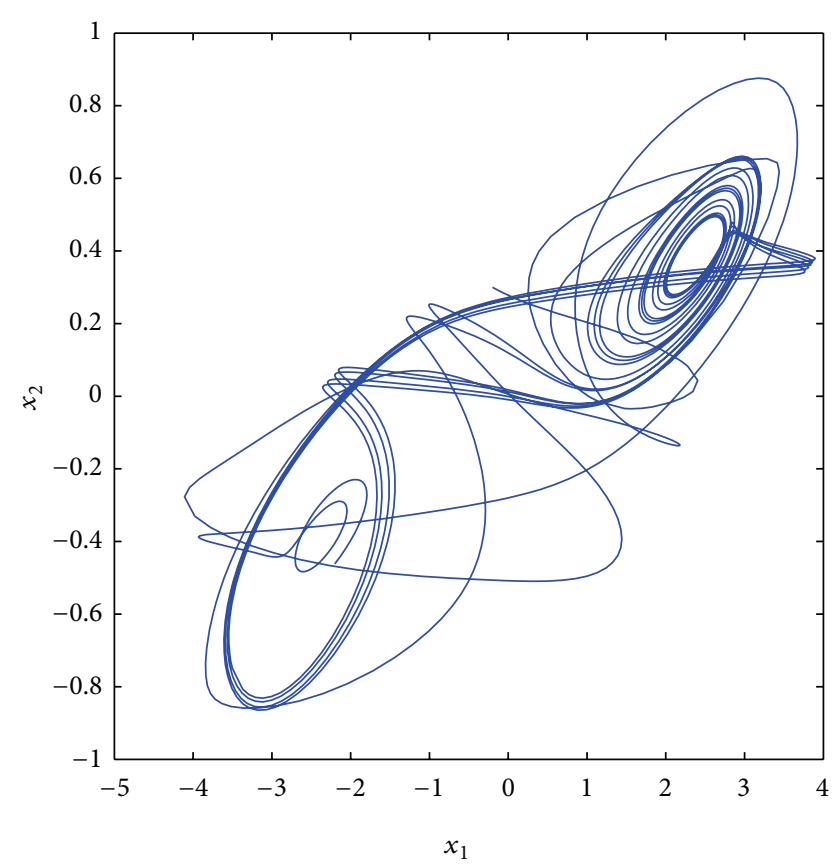

FIGURE 1: The chaotic attractor of the Lu oscillator described by (30) with initial value $x_{1}(s)=0.2, x_{2}(s)=-0.5$, for $s \in[-1,0]$.

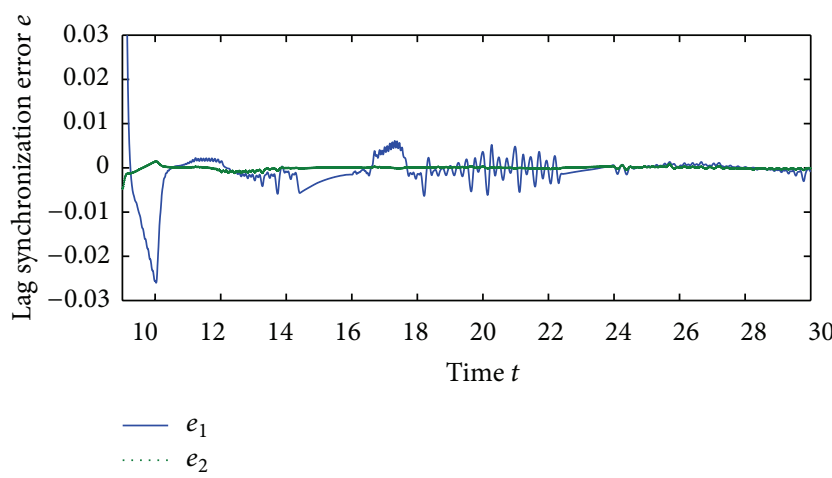

FIGURE 2: Synchronization error curve with $T=8, \sigma=0.8$, and $k=20$.

\section{Conclusions}

In this paper, we have formulated the lag synchronization problem for chaotic neural networks by means of periodically intermittent control. Stochastic synchronization criteria were established based on Lyapunov stability theory and linear matrix inequality techniques. Numerical simulations have showed the validity of theoretical result.

\section{References}

[1] L. M. Pecora and T. L. Carroll, "Synchronization in chaotic systems," Physical Review Letters, vol. 64, no. 8, pp. 821-824, 1990.

[2] L. M. Pecora and T. L. Carroll, "Driving systems with chaotic signals," Physical Review A, vol. 44, no. 4, pp. 2374-2383, 1991.
[3] N. F. Rulkov, M. M. Sushchik, L. S. Tsimring, and H. D. I. Abarbanel, "Generalized synchronization of chaos in directionally coupled chaotic systems," Physical Review E, vol. 51, no. 2, pp. 980-994, 1995.

[4] R. Mainieri and J. Rehacek, "Projective synchronization in three-dimensional chaotic systems," Physical Review Letters, vol. 82, no. 15, pp. 3042-3045, 1999.

[5] M. G. Rosenblum, A. S. Pikovsky, and J. Kurths, "Phase synchronization of chaotic oscillators," Physical Review Letters, vol. 76, no. 11, pp. 1804-1807, 1996.

[6] M. G. Rosenblum, A. S. Pikovsky, and J. Kurths, "From phase to lag synchronization in coupled chaotic oscillators," Physical Review Letters, vol. 78, no. 22, pp. 4193-4196, 1997.

[7] C. Masoller and D. H. Zanette, "Anticipated synchronization in coupled chaotic maps with delays," Physica A, vol. 300, no. 3-4, pp. 359-366, 2001.

[8] A. L. Wu, S. P. Wen, and Z. G. Zeng, "Synchronization control of a class of memristor-based recurrent neural networks," Information Sciences, vol. 183, no. 1, pp. 106-116, 2012.

[9] S. P. Wen and Z. G. Zeng, "Exponential stability analysis of memristor-based recurrent neural networks with time-varying delays," Neurocomputing, vol. 97, pp. 233-240, 2012.

[10] Q. Zhang and J. A. Lu, "Full state hybrid lag projective synchronization in chaotic (hyperchaotic) systems," Physics Letters A, vol. 372, no. 9, pp. 1416-1421, 2008.

[11] E. M. Shahverdiev, S. Sivaprakasam, and K. A. Shore, "Lag synchronization in time-delayed systems," Physics Letters A, vol. 292, no. 6, pp. 320-324, 2002.

[12] C. D. Li, X. F. Liao, and K. W. Wong, "Chaotic lag synchronization of coupled time-delayed systems and its applications in secure communication," Physica D, vol. 194, no. 3-4, pp. 187202,2004

[13] J. Zhou, T. Chen, and L. Xiang, "Chaotic lag synchronization of coupled delayed neural networks and its applications in secure communication," Circuits, Systems, and Signal Processing, vol. 24, no. 5, pp. 599-613, 2005.

[14] S. Boyd, L. El Ghaoui, E. Feron, and V. Balakrishnan, Linear Matrix Inequalities in System and Control Theory, Society for Industrial and Applied Mathematics (SIAM), Philadelphia, Pa, USA, 1994

[15] A. Halanay, Differential Equations: Stability, Oscillations, Time Lags, Academic Press, San Diego, Calif, USA, 1966.

[16] J. J. Huang, C. D. Li, and Q. Han, "Stabilization of delayed chaotic neural networks by periodically intermittent control," Circuits, Systems, and Signal Processing, vol. 28, no. 4, pp. 567579, 2009.

[17] H. T. Lu, "Chaotic attractors in delayed neural networks," Physics Letters A, vol. 298, pp. 109-116, 2002. 


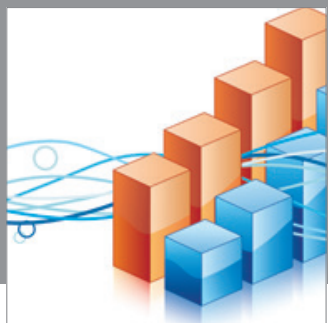

Advances in

Operations Research

mansans

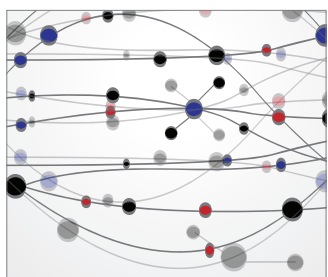

The Scientific World Journal
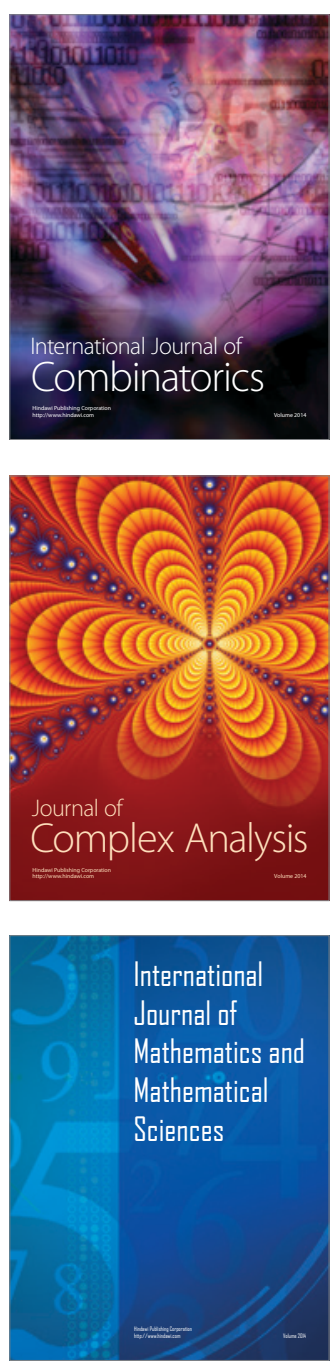
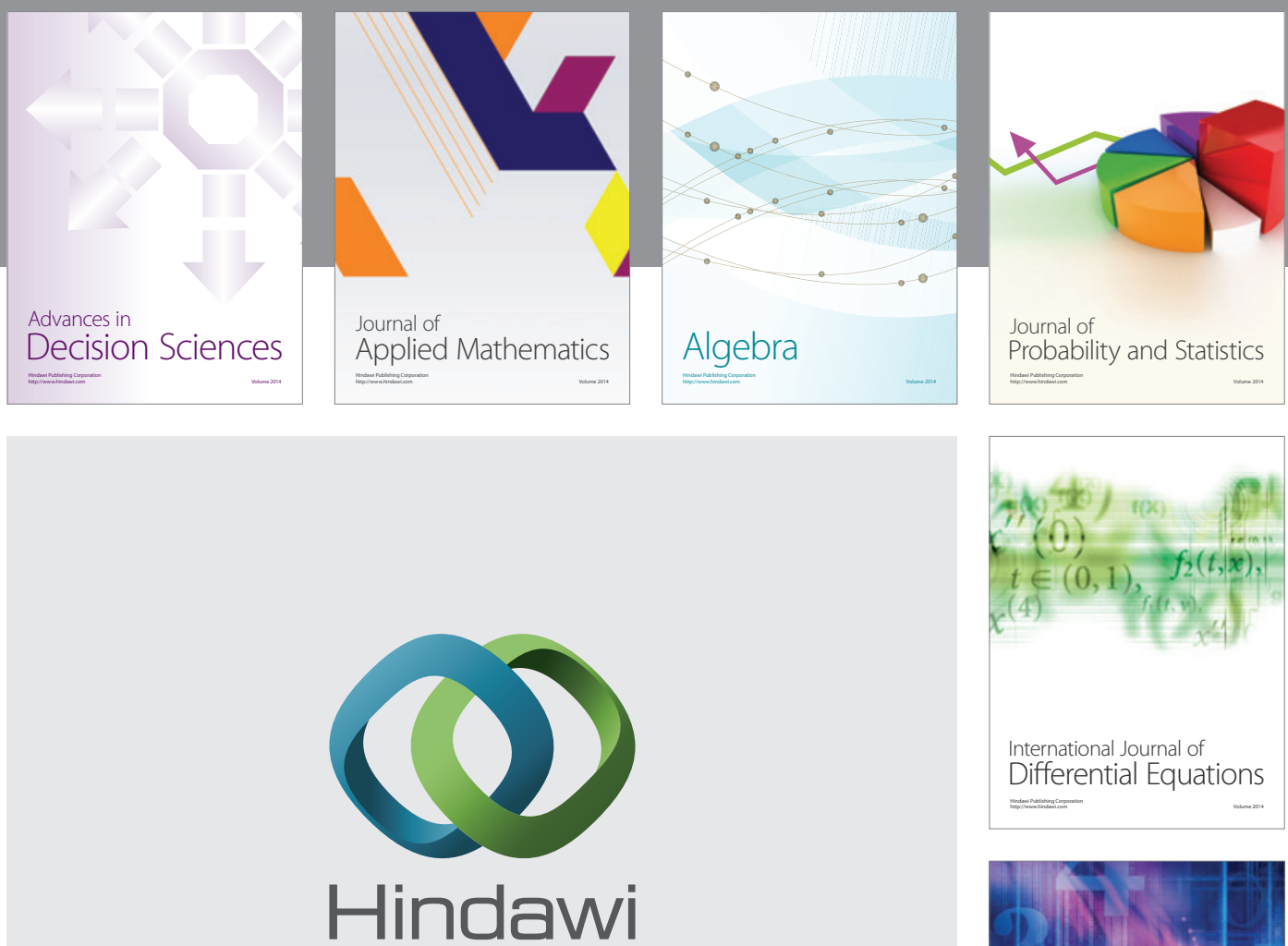

Submit your manuscripts at http://www.hindawi.com
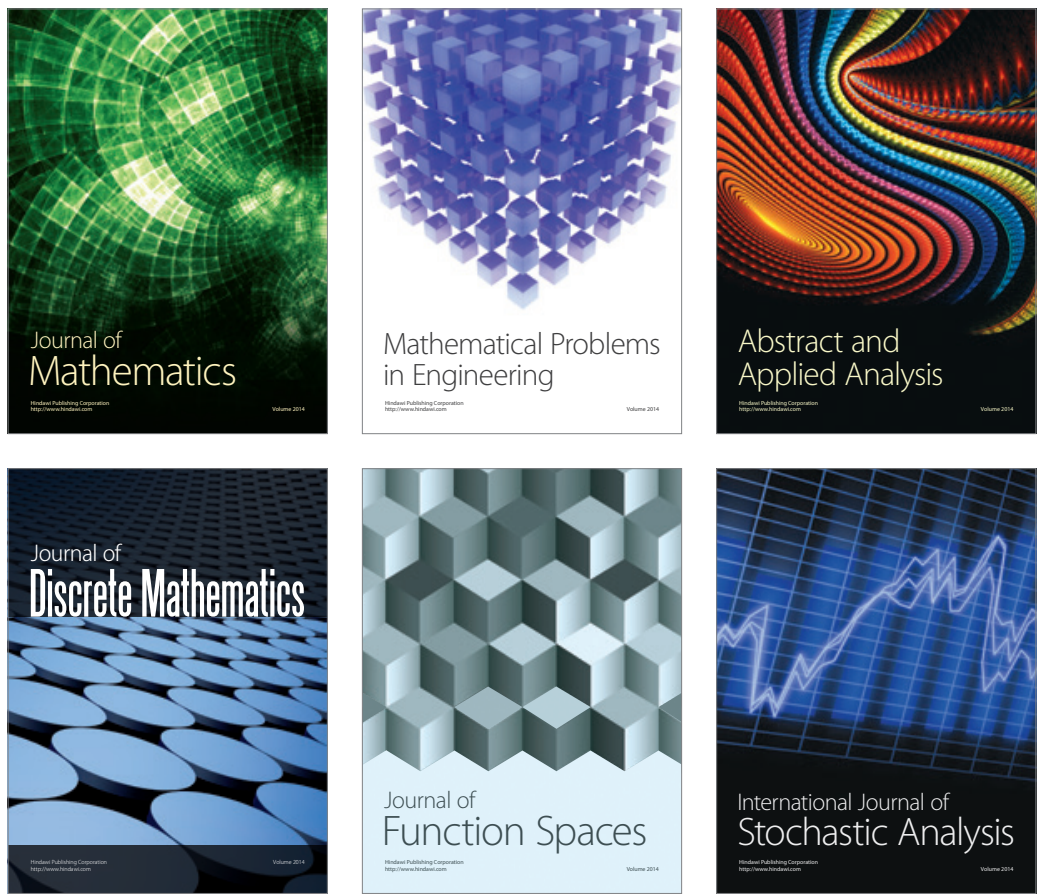

Journal of

Function Spaces

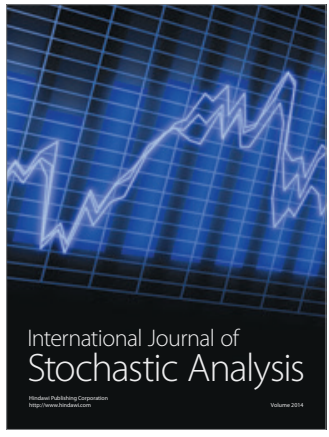

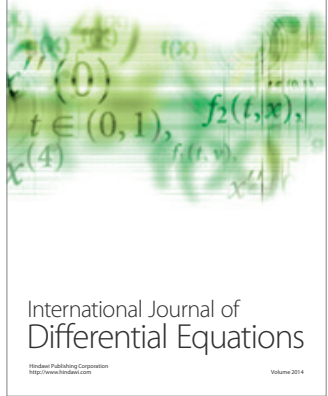
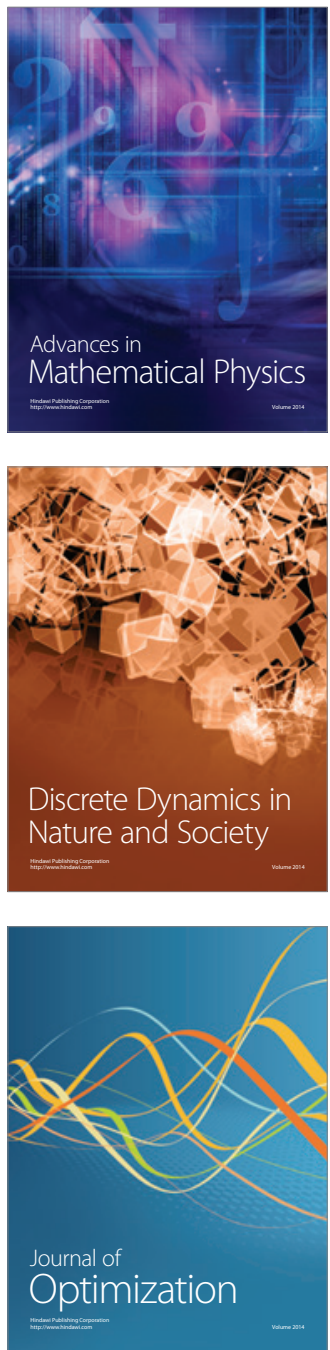